\title{
Hamilton-Jacobi equation and the breaking of the WKB approximation
}

\author{
F. Canfora \\ Università di Salerno, Dipartimento di Fisica "E.R.Caianiello", Istituto Nazionale \\ di Fisica Nucleare, GC di Salerno, Via S.Allende, 84081 Baronissi (Salerno) Italy; \\ e-mail: canfora@sa.infn.it, Phone: +39-089-965228, Fax: +39-089-965275.
}

\begin{abstract}
A simple method to deal with four dimensional Hamilton-Jacobi equation for null hypersurfaces is introduced. This method allows to find simple geometrical conditions which give rise to the failure of the WKB approximation on curved spacetimes. The relation between such failure, extreme blackholes and the Cosmic Censor hypothesis is briefly discussed.
\end{abstract}

Key words: Extreme black holes, WKB approximation PACS: 04.20.Dw, 04.20.-q, 04.70.-s, 11.80.Fv.

\section{Introduction}

Hamilton-Jacobi (henceforth, HJ) equation is one of the most deeply analyzed equation of theoretical physics because of its application in many fields of great interest such as theoretical optics, quantum mechanics, Hamiltonian dynamics. In gravitational physics many important applications are based on HJ equation as, for instance, gravitational lensing. HJ equation also encodes the lightcones structure of space-time and its analysis is also fruitful to disclose the asymptotic symmetries of space-time itself. In blackholes physics also, since the Carter's works [6] [7], HJ equation played a prominent role in clarifying many important local as well as global properties of such interesting objects. A seemingly new method to deal with the curved four dimensional HJ equation seems to be able to disclose interesting relations between complex structures, BPS states in gravity and the breaking of the WKB approximation on curved space-time. In particular, this method discloses in a clear and simple way the relation between the impossibility to adopt the geometric optics approximation on certain curved backgrounds and the presence of naked singularities. 


\section{The method}

In the limit of small wave-lenghts, beams of null geodesics describing, for example, electromagnetic or gravitational waves can be analyzed through the WKB approximation. The rapidly oscillating factor multiplying the slowly varying amplitude of the wave is usually written as $\exp (-i S)$ where $S$ is the eikonal function representing the wave fronts. On a four-dimensional curved manifold $(M, g), S$ satisfies the following HJ equation:

$$
g^{\mu \nu} \partial_{\mu} S \partial_{\nu} S=0
$$

$g^{\mu \nu}$ being the inverse metric. To exploit the quaternionic structure of four dimensional light cones it is convenient to use a non holonomic base:

$$
\begin{aligned}
g & =\eta_{a b} \theta^{a} \theta^{b} \\
\theta^{a} & =\varepsilon_{\mu}^{(a)} d x^{\mu}, g_{\mu \nu}=\eta_{a b} \varepsilon_{\mu}^{(a)} \varepsilon_{\nu}^{(b)} \\
d \theta^{a} & \neq 0
\end{aligned}
$$

where the greek indices $\mu$ run over space-time coordinates, the latin indices $a=0, . ., 3$ run over the tetrad basis $\varepsilon_{\mu}^{(a)}$ and $\eta_{a b}$ is the Minkowski tensor on

tetrad indices. We will denote with $\varepsilon_{(a)}^{\mu}$ the inverse tetrad. In terms of the tetrad components eq. (1) reads:

$$
\left(\varepsilon_{(0)}^{\mu} \partial_{\mu} S\right)^{2}=\sum_{i=1}^{3}\left(\varepsilon_{(i)}^{\mu} \partial_{\mu} S\right)^{2}
$$

Equation (3) can be rewritten in the following useful form:

$$
\operatorname{det} \Theta=0, \Theta=\left\{\begin{array}{cc}
\varepsilon_{(0)}^{\mu} \partial_{\mu} S+\varepsilon_{(1)}^{\mu} \partial_{\mu} S & \varepsilon_{(2)}^{\mu} \partial_{\mu} S+\sqrt{-1} \varepsilon_{(3)}^{\mu} \partial_{\mu} S \\
\varepsilon_{(2)}^{\mu} \partial_{\mu} S-\sqrt{-1} \varepsilon_{(3)}^{\mu} \partial_{\mu} S & \varepsilon_{(0)}^{\mu} \partial_{\mu} S-\varepsilon_{(1)}^{\mu} \partial_{\mu} S
\end{array}\right\} .
$$

It is well known that the determinant of a matrix is zero if and only if either two rows (two columns) are proportional or one row (one column) vanishes.

Let us firstly consider the case in which $S$ is a real function. When the first row vanishes one obtains:

$$
\begin{aligned}
\varepsilon_{(0)}^{\mu} \partial_{\mu} S+\varepsilon_{(1)}^{\mu} \partial_{\mu} S & =0 \\
\varepsilon_{(2)}^{\mu} \partial_{\mu} S+\sqrt{-1} \varepsilon_{(3)}^{\mu} \partial_{\mu} S & =0 .
\end{aligned}
$$

Separating the real and the imaginary part in eq. (6) and rewriting eq. (5) we 
get

$$
\begin{aligned}
\varepsilon_{(2)}^{\mu} \partial_{\mu} S & =0 \\
\varepsilon_{(3)}^{\mu} \partial_{\mu} S & =0 \\
\left(\varepsilon_{(0)}^{\mu}+\varepsilon_{(1)}^{\mu}\right) \partial_{\mu} S & =0 .
\end{aligned}
$$

The above simple system of equations for $S$ tells us that locally $S$ has a non trivial Lie derivative only along the tetrad $\varepsilon_{(0)}^{\mu}-\varepsilon_{(1)}^{\mu}$ so that $S$ can be easily found by solving eq. (9) through the method of characteristic. Eqs. (9), (7) and (8) could be the starting point of the WKB analysis in gravitational lensing, but usually one only considers the characteristics of the above equations, that is, usually only the light rays analysis is performed (see, for example, [19]). It is clear that $S$ carries more physical informations then single light rays, so, as far as gravitational lensing is concerned, this method to treat HJ equation could be useful to obtain global properties of the light beams such as the shear, the curvature of the wavefronts and so on.

When the two rows of $\Theta$ are proportional (the function of proportionality $k$ being an arbitrary non vanishing function on $M$ ) and $S$ is real, the equations for $S$ can be written as follows:

$$
\begin{aligned}
\left(k-\frac{1}{k}\right) \varepsilon_{(0)}^{\mu} \partial_{\mu} S & =\left(k+\frac{1}{k}\right) \varepsilon_{(1)}^{\mu} \partial_{\mu} S \\
2 \varepsilon_{(0)}^{\mu} \partial_{\mu} S & =\left(k+\frac{1}{k}\right) \varepsilon_{(2)}^{\mu} \partial_{\mu} S \\
\varepsilon_{(3)}^{\mu} \partial_{\mu} S & =0 .
\end{aligned}
$$

The above equations, with a suitable choice of $k$, can describe interesting phenomena such as geodesics wrapping round and round in the (2)-(3) direction. It could be rather fruitful to exploit the above method together with an interesting method developed in [12] [13] to deal with the singularities of light cones which can be described in a rather detailed way. Indeed, one of the most important topic in which the above scheme could be an important tools is holography (see, for two detailed reviews, [5] [1]). In the elegant framework of covariant entropy bounds [3] [4] which refined pioneering ideas of Bekenstein [2] 't Hooft [21] and Susskind [20], an important role to deduce the bounds on the entropy of a given two dimensional spacelike surface $\Sigma$ is played by the null hypersurface spanned by the non expanding light rays emanating from $\Sigma$ which, eventually, terminate at caustics. The analysis of such null hypersurfaces is mainly based on geodetics and Raychaudhuri equations. Any null hypersurfaces can be seen as a solution of eq. (1) so it is clear that the method here proposed could be very important in a holographic perspective: to solve eq. (1) is equivalent to solve the null geodetics equation together with Raychaudhuri equation since the wave fronts $S$ gives informations both on the light rays (through its characteristics) and on the shear and on the expansion of the light beams (through the induced geometry of the null hypersurfaces 
$S=$ const which can be easily studied once $S$ is given). The method here proposed, which reduces eq. (1) to a much simpler set of linear partial differential equations, seems to be an effective tool to explore holography.

Now, let us consider the case in which $S$ can be a complex function: $S=$ $V+\sqrt{-1} W$. It is fair to say that, in this case, the WKB approximation breaks down since, with a complex $S$, it is not true anymore that we have a wave with a slowly varying amplitude $A$ and a rapidly varying phase $S=V+$ $\sqrt{-1} W$, being the amplitude multiplied by $\exp (-W)$ which is rapidly varying. The analysis of this case is rather interesting: firstly, it clarifies which is the geometrical structure responsible of the breaking of the WKB approximation. Moreover, it shed new light on some interesting phenomena occurring in the presence of such geometrical structure. A remark is in order here. It is rather trivial to construct special complex solutions of eq. (1): given a real solution of eq. (1) $S_{0}$, the complex function $S_{0}+\sqrt{-1} F\left(S_{0}\right)$ is a complex solution of eq. (1) for any differentiable real function $F(x)$. Obviously, this kind of complex solutions are uninteresting and have no physical meaning. On the contrary, the complex solutions we will search for arise only in very special situations and are related to geometric structures (which generalize the Cauchy-Riemann conditions) signaling interesting physical phenomena.

When one rows of $\Theta$ vanishes, separating the real and the imaginary part one obtains the following linear system of equations for $V$ and $W$ :

$$
\begin{aligned}
\left(\varepsilon_{(0)}^{\mu} \pm \varepsilon_{(1)}^{\mu}\right) \partial_{\mu} V & =0, \quad\left(\varepsilon_{(0)}^{\mu} \pm \varepsilon_{(1)}^{\mu}\right) \partial_{\mu} W=0 \\
\varepsilon_{(3)}^{\mu} \partial_{\mu} W & =\mp \varepsilon_{(2)}^{\mu} \partial_{\mu} V, \quad \varepsilon_{(2)}^{\mu} \partial_{\mu} W= \pm \varepsilon_{(3)}^{\mu} \partial_{\mu} V .
\end{aligned}
$$

where $\varepsilon_{(0)}^{\mu} \pm \varepsilon_{(1)}^{\mu}$ is the propagation direction of the geodesic flows and both the real and the imaginary part of $S$ have vanishing Lie derivative along the propagation direction. The non trivial dependence in the transversal directions is parametrized by a sort of generalized harmonic functions in the (2) - (3) directions since eqs. (14) can be thought as generalized Cauchy-Riemann conditions for $V$ and $W$. To see this, let us consider the case in which

$$
\begin{aligned}
\varepsilon_{(2)}^{\mu} & =C \partial_{2}+D \partial_{3}, \quad \varepsilon_{(3)}^{\mu}=E \partial_{2}+F \partial_{3} \\
C F-D E & \neq 0 .
\end{aligned}
$$

With the above tetrads, eqs. (14) read:

$$
\begin{aligned}
\partial_{i} W & =\widetilde{T}_{i}^{j} \partial_{j} V, \quad i, j=2,3 \\
\widetilde{T}_{j}^{i} & =\frac{1}{C F-D E}\left(\begin{array}{cc}
E F+C D & F^{2}+C^{2} \\
-\left(E^{2}+C^{2}\right)-(E F+C D)
\end{array}\right),
\end{aligned}
$$

thus, we see that an important role in order to have complex solutions is played by the tensor defined in eq. (16) being a sort of complex structure 
in the transversal directions spanned by $\varepsilon_{(2)}^{\mu}$ and $\varepsilon_{(3)}^{\mu}$. In the following, some geometrical conditions on eqs. (14) ensuring the possibility to have non trivial complex solutions of HJ equation together with two interesting examples will be analyzed.

In the case in which the two rows are proportional the equations read:

$$
\begin{aligned}
\varepsilon_{(0)}^{\mu} \partial_{\mu} S+\varepsilon_{(1)}^{\mu} \partial_{\mu} S & =k_{C}\left(\varepsilon_{(2)}^{\mu} \partial_{\mu} S-\sqrt{-1} \varepsilon_{(3)}^{\mu} \partial_{\mu} S\right) \\
\varepsilon_{(2)}^{\mu} \partial_{\mu} S+\sqrt{-1} \varepsilon_{(3)}^{\mu} \partial_{\mu} S & =k_{C}\left(\varepsilon_{(0)}^{\mu} \partial_{\mu} S-\varepsilon_{(1)}^{\mu} \partial_{\mu} S\right)
\end{aligned}
$$

$k_{C}=A+\sqrt{-1} B$ being a non zero complex function on $M$. Eqs. (17) and (18) can be splitted into four real equations as follows:

$$
\begin{aligned}
L_{(b)}^{(a)} X_{(a)} & =Y_{(b)}, \quad X_{(a)}=\varepsilon_{(a)}^{\mu} \partial_{\mu} W, \quad Y_{(a)}=N_{(a)}^{(b)} \varepsilon_{(b)}^{\mu} \partial_{\mu} V, \\
L_{(b)}^{(a)} & =\left(\begin{array}{cccc}
0 & 0 & -B & A \\
1 & 1 & -A & -B \\
-B & B & 0 & 1 \\
A & -A & -1 & 0
\end{array}\right), \quad N_{(b)}^{(a)}=\left(\begin{array}{cccc}
1 & 1 & -A & -B \\
0 & 0 & B & -A \\
-A & A & 1 & 0 \\
-B & B & 0 & 1
\end{array}\right)
\end{aligned}
$$

To express the imaginary part of $S$ in terms of the real part, we have to solve the linear system (19) for the $X_{(a)}$. However, $\operatorname{det} L=0$ since only three rows are independent and the first row $\vec{r}_{(1)}$ is a linear combination of the third $\vec{r}_{(3)}$ and the fourth $\vec{r}_{(4)}$ rows $\vec{r}_{(1)}=A \vec{r}_{(3)}+B \vec{r}_{(4)}$. In order for the system of equations (19) to be compatible, the following relation, which determines the real part of $S$, must hold:

$$
\begin{gathered}
Y_{(0)}=A Y_{(2)}+B Y_{(3)} \Rightarrow \\
\frac{\left(A^{2}+B^{2}-1\right)}{\left(A^{2}+B^{2}+1\right)} \varepsilon_{(1)}^{\mu} \partial_{\mu} V+\frac{2 A}{\left(A^{2}+B^{2}+1\right)} \varepsilon_{(2)}^{\mu} \partial_{\mu} V+ \\
+\frac{2 B}{\left(A^{2}+B^{2}+1\right)} \varepsilon_{(3)}^{\mu} \partial_{\mu} V=\varepsilon_{(0)}^{\mu} \partial_{\mu} V .
\end{gathered}
$$

By standard linear algebra's consideration, when the above equation is satisfied, we can discard the first equation of the system (19) and freely assign $\varepsilon_{(0)}^{\mu} \partial_{\mu} W$, so we will take $\varepsilon_{(0)}^{\mu} \partial_{\mu} W=0$. Eventually, it is convenient, in the reduced system (19), to express the Lie derivative of $V$ along the timelike tetrad $\varepsilon_{(0)}^{\mu} \partial_{\mu} V$ in terms of the Lie derivative along the spacelike ones $\varepsilon_{(i)}^{\mu} \partial_{\mu} V$ (with $(i)=1,2,3)$ through eq. (20). Thus, we arrive at the following system in which 
only spacelike Lie derivatives are involved:

$$
\begin{aligned}
L_{(j)}^{(i)} X_{(i)} & =\widetilde{N}_{(j)}^{(i)} \varepsilon_{(i)}^{\mu} \partial_{\mu} V, \quad L_{(j)}^{(i)}=\left(\begin{array}{ccc}
0 & A & B \\
B & 0 & 1 \\
-A & -1 & 0
\end{array}\right), \quad \operatorname{det} L_{(j)}^{(i)} \neq 0, \\
\widetilde{N}_{(j)}^{(i)} & =\left(\begin{array}{ccc}
0 & B & A \\
\frac{2 A}{A^{2}+B^{2}+1} & 1-\frac{2 A^{2}}{A^{2}+B^{2}+1} & -\frac{2 A B}{A^{2}+B^{2}+1} \\
\frac{2 B}{A^{2}+B^{2}+1} & -\frac{2 A B}{A^{2}+B^{2}+1} & 1-\frac{2 B^{2}}{A^{2}+B^{2}+1}
\end{array}\right) .
\end{aligned}
$$

The above equation can be formally solved for $X_{(i)}$ as follows:

$$
X_{(i)}=\varepsilon_{(i)}^{\mu} \partial_{\mu} W=\left(L^{-1} \circ \widetilde{N}\right)_{(i)}^{(j)} \varepsilon_{(j)}^{\mu} \partial_{\mu} V .
$$

Now, to have a clearer geometrical picture, let us consider an holonomic coordinates system in which the spacelike vector fields of the tetrad basis $\varepsilon_{(i)}^{\mu}$ have no timelike components: $\varepsilon_{(i)}^{0}=0, \forall i=1, . ., 3$. This, locally, can always be achieved. In this coordinates system, the components of the spacelike vector fields of the tetrad basis $\varepsilon_{(j)}^{\alpha}$ (where we use the first greek letters $\alpha, \beta, \gamma$ and so on, to denote spacelike indices) can be seen as an invertible map between spacelike coordinates vector fields $\partial_{\alpha}$ and the spacelike tetrad vector fields. From eq. (21), we can express $\partial_{\alpha} W$ as follows:

$$
\begin{aligned}
\partial_{\alpha} W & =T_{\alpha}^{\beta} \partial_{\beta} V \\
T_{\alpha}^{\beta} & =\left(\varepsilon_{(i)}^{\alpha}\right)^{-1}\left(L^{-1} \circ \widetilde{N}\right)_{(i)}^{(j)} \varepsilon_{(j)}^{\beta}
\end{aligned}
$$

where $T_{\beta}^{\alpha}$ is a tensor field depending only on the metric of the space-time and on $A$ and $B$. Eq. (22) (as well as eq. (15) in the previous case) is related to the range of applicability of the standard WKB approximation.

Let $V$ be a real solution of HJ equation (with suitable choices of $k^{1}$ solutions of eqs. (10), (11) and (12) are solutions of eq. (20) too), if eq. (22) and eq. (15) can be fulfilled with reasonable boundary conditions, then eq. (22) generates a new solution with the same real part and an imaginary part representing a geometrical magnification or damping factor. Thus, in these cases, the WKB approximation cannot be used anymore because the amplitudes of the waves travelling in such regions are multiplied by rapidly varying magnification factors of the form $\exp (-W)$.

The problem is to determine the geometrical properties of $T_{\beta}^{\alpha}$ that make this possible. Clearly, not any tensor makes eq. (22) (or eq. (15)) consistent: we

$\overline{1}$ For example $k=1, B=0$ and $A=1$. 
have to impose that $\partial_{\alpha \gamma}^{2} W=\partial_{\gamma \alpha}^{2} W$ :

$$
\partial_{\alpha \gamma}^{2} W=\partial_{\gamma \alpha}^{2} W \Rightarrow \partial_{\gamma}\left(T_{\alpha}^{\beta} \partial_{\beta} V\right)=\partial_{\alpha}\left(T_{\gamma}^{\beta} \partial_{\beta} V\right)
$$

The solvability of eqs. (24) and the similar compatibility conditions deduced from eq. (14) with reasonable boundary conditions puts strong constraint on the topology. Let us consider a simple but interesting example, four dimensional PP-waves (first introduced in [17] [18]):

$$
\begin{aligned}
g & =d t^{2}-d z^{2}+H(t-z, x, y) d(t-z)^{2}-d x^{2}-d y^{2}, \\
\left(\partial_{x}^{2}+\partial_{y}^{2}\right) H & =0 .
\end{aligned}
$$

In this case, eqs. (14), which allows the arising of a complex solution $W$, become:

$$
\partial_{y} W=-\partial_{x} V, \quad \partial_{x} W=\partial_{y} V
$$

This Cauchy-Riemann conditions simply tell us that $V$ is a two-dimensional harmonic function and $W$ is the harmonic function conjugated to $V$. However, when one imposes the physical boundary condition that $V$ has to approach to a constant in the limit $x^{2}+y^{2} \rightarrow \infty$, one only finds the trivial solution because of the maximum principle for harmonic functions. In the case of singular PPwaves, (which are naturally coupled to cosmic string along the $z$-axis and/or $\gamma$-ray bursts propagating in the same direction [9]) the metric and the Einstein equations reduce to:

$$
\begin{aligned}
g & =d t^{2}-d z^{2}+H(t-z, x, y) d(t-z)^{2}-d x^{2}-d y^{2}, \\
\left(\partial_{x}^{2}+\partial_{y}^{2}\right) H & =\sum^{N} \mu_{i} \delta_{i}\left(x_{i}(t-z), y_{i}(t-z)\right)
\end{aligned}
$$

where $N$ is the number of cosmic strings, $\mu_{i}$ is the energy per unit of length and $\left(x_{i}(t-z), y_{i}(t-z)\right)$ is the position of the $i$-th cosmic string in the $x-y$ plane. Even if eqs. (14) remain formally the same as eqs. (25), now there are singularities in the $x-y$ plane corresponding to the positions of the cosmic strings. Thus, harmonic functions approaching to a constant in the limit $x^{2}+$ $y^{2} \rightarrow \infty$ in this case exist thanks to the singularities in the $x-y$ plane and non trivial complex solutions of the HJ equation appear. Another interesting example is the Reissner-Nordstrom blackhole:

$$
g=\left(1-\frac{2 M}{r}+\frac{Q^{2}}{r^{2}}\right) d t^{2}-\left(1-\frac{2 M}{r}+\frac{Q^{2}}{r^{2}}\right)^{-1} d r^{2}-r^{2} d \Omega^{2}
$$

In the case in which the 2 and 3 directions are $r$ and $\varphi$ and $\theta=\pi / 2$, eqs. (14) are:

$$
\begin{gathered}
\Gamma \partial_{r} W=-\partial_{\varphi} V, \quad \partial_{\varphi} W=\Gamma \partial_{r} V \Rightarrow \\
{\left[\Gamma \partial_{r}^{2}+\frac{1}{\Gamma} \partial_{\varphi}^{2}+\left(\partial_{r} \Gamma\right) \partial_{r}\right] V=0, \quad \Gamma=r\left(1-\frac{2 M}{r}+\frac{Q^{2}}{r^{2}}\right)^{1 / 2}}
\end{gathered}
$$


If $M>|Q|$, (in which case the surface gravity is non vanishing) the operator appearing in eq. (27) is of mixed type: elliptic outside the two zeros of $g_{t t}$ and hyperbolic inside. In this case, there is no real continuous piece-wise smooth solution of eq. (27) approaching to zero for $r \rightarrow \infty$ (this can be rigorously proved using standard techniques in linear partial differential equations). However, if $M=|Q|$ (so that the surface gravity is vanishing), the situation changes and real non trivial continuous (but not differentiable on the line $r=M$ ) solutions appear since the operator in eq. (27) is elliptic degenerate on the line $r=M$. The detailed proof of this fact is trivial but a little bit long. It is possible to convince oneself of this by analyzing the Majumdar-Papapetrou solution [14] [16] which represents $N$ extreme charged blackholes in equilibrium and is a BPS states in supergravity [15]. The metric (26) reads:

$$
\begin{aligned}
g & =U^{2} d t^{2}-U^{-2}\left(d x^{2}+d y^{2}+d z^{2}\right) \\
\left(\partial_{x}^{2}+\partial_{y}^{2}+\partial_{z}^{2}\right) U & =\sum^{N} M_{i} \delta\left(x_{i}, y_{i}, z_{i}\right)
\end{aligned}
$$

where $\left(x_{i}, y_{i}, z_{i}\right)$ is the position of the $i$-th blackhole and $M_{i}$ its mass equal to its charge. In the case in which the 2 and 3 directions are $x$ and $y$ and $z=z_{i}$, eqs. (14) look like eqs. (25), but there is a singularity in the $x-y$ plane corresponding to the positions of the $i$-th blackhole $\left(x_{i}, y_{i}\right)$. Thus, non trivial harmonic functions approaching to a constant for $x^{2}+y^{2} \rightarrow \infty$ appear. Hence, extreme blackholes admit non trivial complex solutions of the HJ equation. This characteristic of extreme charged blackholes is indeed related to their BPS nature. Non trivial complex solutions appear in the extreme case because the two dimensional operator in eq. (27) is elliptic degenerate on the line $r=M$. This happens because the components $g_{t t}$ of the metric tensor (which approaches to 1 for large $r$ ) has only one zero and does not change sign and this is a consequence of the fact that extreme charged blackholes admit a globally defined timelike Killing vector field ${ }^{2}$. The existence of a global timelike Killing vector field is a necessary condition in order to have a BPS states in supergravity (see the discussion of Gibbons in [15]). It is a well known phenomenon (see, for example, [15] and references therein) that BPS states in field theory often carry complex structures similar to the ones in eq. (22) and eq. (14). Eventually, when $M<|Q|$, the operator appearing in eq. (27) is elliptic non degenerate and, for any given Dirichlet boundary condition, the Cauchy problem admits a unique solution. It is worth to note that the problem to understand if a given gravitational field does or does not represent an extreme blackhole is reduced to the analysis of Cauchy problems for two dimensional linear partial differential equations, this can be a great simplification in more general cases. We learn from this examples that the solvability of eqs. (14) and eqs. (22) is able to detect space-time region with not only a

2 For example, in the Schwarzschild case, the Killing vector $\partial_{t}$ is timelike outside the horizon and spacelike inside. 
strong gravitational field but also strong electromagnetic fields and/or angular momenta.

The solvability of eqs. (14), (15) and (22) in asymptotically flat space-times and, in particular, its character could be related to the surface gravity of the horizon in the general case which, unfortunately, is very difficult to analyze. The following argument provides an explanation of this relation in the stationary case in which the event horizon is a Killing horizon. It is well known that if the surface gravity is non vanishing, the Killing horizon (which can be thought as a real solution of HJ equation) is bifurcating while, when it vanishes, there is no bifurcation (see, for example, [8]). However, it should be kept in mind that, when one says "there is no bifurcation if the surface gravity vanishes" one usually means that there is no real bifurcation. In fact, when the surface gravity approaches to zero the bifurcation phenomenon does not disappear, the new solution simply becomes complex and eq. (24) tells us precisely when such complex solutions appear. This explains why the solvability of eqs. (15) and (22), through eq. (24), is able to detect Killing horizons with vanishing surface gravity, that is, extreme black holes.

For the above reasons, there is also a relation with the problem of the Cosmic Censor conjecture. A Cosmic Censor should not allow gravitational fields with generic breaking of the WKB approximation, which characterizes extreme blackholes, while he should allow only gravitational fields for which eqs. (14) and eqs. (22) can not be satisfied, in such a way that gravitational fields would not go beyond extremality. These arguments shows that a deeper analysis of HJ equation with the above method could shed light on the physical constraints needed to make Cosmic Censor conjecture true.

\section{Conclusions}

In this paper, a new method to deal with HJ equation for null hypersurfaces in curved four dimensional space-times has been presented. This method, based on a convenient form for HJ equation, simplifies the HJ equation without any reference to the field equations for the gravitational field, so that it could be used even in theories other then general relativity. It allows to find explicitly the geometrical conditions on the metric which the gravitational field has to satisfies in order to have non trivial complex solutions of the HJ equation. This space-times, in which the WKB approximation cannot be used, are of great importance since the origin of the breaking of the WKB scheme is related to the presence of extreme blackholes. Thus, the analysis of the case in which such non trivial complex solutions arise could also shed light on the physical conditions to be imposed in order to make the Cosmic Censor conjecture true. 


\section{References}

[1] O. Aharony, S. S. Gubser, J. Maldacena, H. Ooguri, Y. Oz, "Large N Field Theories, String Theory and Gravity" Phys. Rept. 323, 183 (2000) and references therein.

[2] J. D. Bekenstein, "Universal Upper Bound on the Entropy-to-Energy Ratio for Bounded Systems" Phys. Rev. D 23, 287 (1981).

[3] R. Bousso, "Holography in General Space-Times" JHEP 9906, 028 (1999).

[4] R. Bousso, " A Covariant Entropy Conjecture" JHEP 9907, 004 (1999).

[5] R. Bousso, "The Holographic Principle" Rev. Mod. Phys. 74, 825 (2002) and references therein.

[6] B. Carter, "Global Structure of the Kerr Family of Gravitational Fields" Phys. Rev. 174, 1559 (1968).

[7] B. Carter, "Hamilton-Jacobi and Schrodinger separable solutions of Einstein's equations" Comm. Math. Phys. 10, 280 (1968).

[8] S. Chandrasekar, The mathematical theory of black holes, (Clarendon Press, Oxford, 1983).

[9] F.Canfora, G.Vilasi, Phys. Lett. B 585,"Spin-1 Gravitational Waves and Their Natural Sources" 193 (2004).

[10] F. Canfora, G. Vilasi, P. Vitale, "Nonlinear Gravitational Waves and Their Polarization" Phys. Lett. B 545, 373 (2002).

[11] F. Canfora, G. Vilasi, P. Vitale, "Spin-1 Gravitational Waves" gr-qc/021202, to appear on Int. J. Mod. Phys. B.

[12] S. Frittelli, E. T. Newman, G. Silva-Ortigoza, " The Eikonal Equation in Flat Space: Null Surfaces and their Singularities. I" J. Math. Phys. 40, 383 (1999).

[13] S. Frittelli, E. T. Newman, G. Silva-Ortigoza, "The Eikonal Equation in Asymptotically Flat Space-Times" J. Math. Phys. 40, 1041 (1999).

[14] S. D. Majumdar, "A Class of Exact Solutions of Einstein's Field Equations" Phys. Rev. 72, 390 (1947).

[15] D. I. Olive, P. C. West editors, Duality and Supersymmetric Theories, (Cambridge University Press, 1999).

[16] A. Papapetrou, "A Static Solution of the Equations of the Gravitational Fields for an Arbitrary Charge Distribution" Proc. Roy. Irish Acad. A 51, 191 (1947).

[17] A. Peres, "Some Gravitational Waves" Phys. Rev. Lett. 3, 571 (1959).

[18] A. Peres, "Null Electromagnetic Fields in General Relativity Theory" Phys. Rev. 118, 1105 (1960). 
[19] P. Schneider, J. Ehlers, E. E. Falco, Gravitational Lenses, Springer, Berlin, 1992.

[20] L. Susskind, " The World as a Hologram" J. Math. Phys 36, 6377 (1995).

[21] G. 't Hooft, "Dimensional Reduction in Quantum Gravity" gr-qc/9310026. 